\title{
Symmetry reduction in multiband Hamiltonians for semiconductor quantum dots: The role of interfaces and higher energy bands
}

\author{
Stanko Tomić ${ }^{1}$ and Nenad Vukmirović 2 ,a) \\ ${ }^{1}$ Joule Physics Laboratory, School of Computing, Science and Engineering, University of Salford, \\ Salford M5 4WT, United Kingdom \\ ${ }^{2}$ Scientific Computing Laboratory, Institute of Physics Belgrade, University of Belgrade, Pregrevica 118, \\ 11080 Belgrade, Serbia
}

(Received 17 April 2011; accepted 25 July 2011; published online 12 September 2011)

\begin{abstract}
The role of interfaces and higher bands on the electronic structure of embedded semiconductor quantum dots (QDs) was investigated. The term in the multiband $\mathbf{k} \cdot \mathbf{p}$ Hamiltonian that captures the effect of interface band mixing was derived starting from the microscopic theory. It was shown, analytically and numerically, that, with such a term included, the right symmetry of the QD system can be captured. It leads to splitting of otherwise degenerate energy levels of the order of several $\mathrm{meV}$. The inclusion of additional higher bands beyond the ones from the standard eight-band model also leads to the reduction of symmetry from an artificially high one to the true atomistic symmetry of the system, however their quantitative effect is weaker. These results prove that the multiband k.p Hamiltonians are fully capable of describing the correct symmetry of a QD. (C) 2011 American Institute of Physics. [doi:10.1063/1.3631048]
\end{abstract}

\section{INTRODUCTION}

Semiconductor quantum dots (QDs) are highly interesting physical systems both as a platform for fundamental studies of interaction of charges, spins and photons on the nanoscopic scale, ${ }^{1-3}$ and due to various possible applications. ${ }^{4-16}$ While certain physical effects in QDs can be qualitatively understood solely based on discreteness of energy levels or using simple particle-in-a-box models, for most purposes an accurate treatment of the electronic structure of QDs is necessary. Typical self-assembled QDs have millions of atoms and therefore the methods based on $a b$ initio density functional theory, the workhorse of modern electronic structure calculations, are far beyond the reach of present day computational capabilities. ${ }^{17}$ All the methods developed so far are therefore to a lesser or larger extent semiempirical. ${ }^{18-30}$ In the multiband envelope function methods (including the $\mathbf{k} \cdot \mathbf{p}$ method) the system Hamiltonian is represented through a matrix of operators that act upon a vector of envelope functions of several bands. Such a representation where all the relevant quantities are slowly varying continuous functions may lead one to believe that the model is a continuous one and that it cannot capture the full symmetry of the nanostructure. ${ }^{31}$

The multiband $\mathbf{k} \cdot \mathbf{p}$ Hamiltonians ${ }^{32-49}$ are capable of reproducing the bulk band structure more accurately than the standard 8-band Hamiltonian. Some of these, that include a large number of bands ( $\gtrsim 15$ or 30 after incorporation of the spin degree of freedom), are even capable of reproducing the bulk band structure throughout the whole Brillouin zone. Unfortunately, these Hamiltonians have been rarely applied to nanostructures and have not been applied to QDs at all.

\footnotetext{
a) Author to whom correspondence should be addressed. Electronic mail: nenad.vukmirovic@ipb.ac.rs.
}

The effect of interface band mixing ${ }^{50-54}$ has also so far been analyzed only for a single interface or a quantum well structure. There is even a lack of appropriate framework for a description of the effect of interface induced band mixing in quantum dot heterostructures. The goal of this work is to explore the effects of higher bands and interfaces on the electronic structure of QDs.

The most widely used form of the multiband Hamiltonians for the description of III-V material nanostructures with zinc blende crystal structure is the 8-band $\mathbf{k} \cdot \mathbf{p}$ Hamiltonian. ${ }^{55,56}$ This Hamiltonian indeed yields a higher symmetry of the nanostructure than the true atomistic one. When one is not interested in very fine details of the electronic structure, such a shortcoming can be converted into a strength from the computational point of view, as the existing symmetry (not necessarily the correct one) can be exploited to block-diagonalize the Hamiltonian and therefore reduce the computational time. ${ }^{57-60}$ There is however a widespread belief ${ }^{61}$ that the mentioned deficiency is a general feature of multiband $\mathbf{k} \cdot \mathbf{p}$ Hamiltonians and that these cannot be used if one needs to capture the true symmetry of the nanostructure. If this were true, this would be indeed a serious issue. For example, models with high symmetry lead to degeneracies of eigenstates that would be otherwise split and as a consequence lead to inaccuracies in the prediction of polarization dependence of interaction with electromagnetic radiation or the distribution of the excitonic state population and their dynamics.

We find no reason that the mentioned shortcomings of the 8-band k.p Hamiltonian would manifest also in multiband $\mathbf{k} \cdot \mathbf{p}$ Hamiltonians in general. In this work, we therefore analyze the k.p Hamiltonians beyond the standard 8-band one, which either include the effect of higher energy bands or include the effect of interface induced band mixing. For the latter, we derive the analytical form of the appropriate Hamiltonian for all interfaces present in the model QD structure and use it in the calculation to understand its role both 
qualitatively and quantitatively. We show both analytically and numerically that beyond 8-band Hamiltonians are capable of capturing the correct atomistic symmetry of QDs and quantify their effect on the electronic structure of QDs.

\section{METHODS}

As a model system, we consider a QD in the shape of a square-based pyramid, with base to height ratio $b / h=2$, which is the most conventional shape assumed in previous theoretical considerations. ${ }^{18,19,26,29,62}$ The coordinate system is chosen so that vertices of the pyramid are at the points $(b / 2,-b / 2,0)$, $(b / 2, b / 2,0),(-b / 2, b / 2,0),(-b / 2,-b / 2,0)$, and $(0,0, h)$.

The k.p Hamiltonians discussed in this paper have a general form:

$$
H_{\mathbf{k} \cdot \mathbf{p}}=H_{\mathbf{k}}+H_{\mathbf{s o}}+H_{\epsilon}+H_{\mathbf{p z}}+H_{\text {if }}
$$

where $H_{\mathrm{k}}$ is the kinetic part, $H_{\mathrm{so}}$ is the part arising from spinorbit interaction, $H_{\epsilon}$ is the strain part, $H_{\mathrm{pz}}$ is the piezoelectric part of the Hamiltonian that depends on strain, and $H_{\text {if }}$ describes the interface induced band-mixing contribution to the Hamiltonian $H_{\mathbf{k} \cdot \mathbf{p}}$. To identify the effect of the Hamiltonians beyond the standard 8-band one, we have analyzed our model system using several different Hamiltonians that include only certain terms from Eq. (1), each with a different level of sophistication.

(a) The 8-band $\mathbf{k} \cdot \mathbf{p}$ Hamiltonian consisting of the kinetic part only (without the spin-orbit interaction and strain).

(b) The 8-band k.p Hamiltonian consisting of the kinetic part with the spin-orbit interaction taken into account (but without strain).

(c) The 8-band $\mathbf{k} \cdot \mathbf{p}$ Hamiltonian consisting of the kinetic part with the interface band-mixing effects taken into account (but without spin-orbit interaction and strain).

(d) The standard 8-band k·p Hamiltonian consisting of the kinetic part with the spin-orbit interaction and strain, as well as the strain-induced piezoelectric potential. It was assumed that piezoelectric polarization depends linearly on strain, though there are some recent suggestions that quadratic effects should be included as well. ${ }^{16,63,64}$

(e) The 8-band $\mathbf{k} \cdot \mathbf{p}$ Hamiltonian consisting of the kinetic part with the spin-orbit interaction and strain, as well as the strain-induced piezoelectric potential and the interface Hamiltonian.

(f) The 14-band k·p Hamiltonian consisting of the kinetic part only (without the spin-orbit interaction and strain).

(g) The 16-band k·p Hamiltonian consisting of the kinetic part only (without the spin-orbit interaction and strain).

(h) The 14-band k.p Hamiltonian consisting of the kinetic part with the spin-orbit-interaction and strain (as well as the strain-induced piezoelectric potential).

(i) The 16-band $\mathbf{k} \cdot \mathbf{p}$ Hamiltonian consisting of the kinetic part with the spin-orbit-interaction and strain (as well as the strain-induced piezoelectric potential).

The explicit forms of the multiband Hamiltonians used in this work and the corresponding material parameters are given in Sec. I of the supplementary information. ${ }^{65}$
The kppw code, ${ }^{30,59}$ appropriately extended to treat the multiband Hamiltonians and the effect of interfaces, was used for all electronic structure calculations presented. Whenever possible, the symmetry of the Hamiltonian was exploited to reduce the computational effort, as described in our previous work. ${ }^{57-59}$ It is in particular important to note that our numerical implementation in a plane wave basis does not reduce the symmetry of the problem; therefore, all energy level splittings that we report originate from the Hamiltonian itself and not from numerical artifacts. In all calculations where strain is taken into account, it has been modeled using the continuum mechanical model. ${ }^{26,66-70}$

\section{INTERFACE TERM IN K.P HAMILTONIAN}

In the derivation of the interface-induced band mixing terms in the 8-band k'p Hamiltonian applied to our model QD system, we follow the approach of Foreman outlined in Ref. 54. The envelope function Hamiltonian at a point $\mathbf{R}$ in space (in the absence of strain and spin-orbit interaction) is equal to ${ }^{54}$

$$
H_{m n}(\mathbf{R})=\frac{\hbar^{2} k^{2}}{2 m_{0}} \delta_{m n}+\frac{\hbar}{m_{0}} \mathbf{k} \cdot \mathbf{p}_{m n}+\left[u_{m}\left|H_{\mathbf{a}}\right| u_{n}\right]_{\mathbf{R}} .
$$

In this equation, $u_{m}$ and $u_{n}$ are the periodic Bloch functions of band $m$ and $n$ of a bulk reference crystal, $H_{\mathrm{a}}=p^{2} / 2 m_{0}+V(\mathbf{r})$ is the microscopic Hamiltonian that we model using the local empirical pseudopotentials, the square brackets denote the averaging over a unit cell centered on $\mathbf{R}, \mathbf{k}=-i \nabla$ and $\mathbf{p}_{m n}=\left[u_{m}|\mathbf{p}| u_{n}\right]$, where $\mathbf{p}$ is the momentum operator.

The term $\left[u_{m}|V| u_{n}\right]_{\mathbf{R}}$ in the Hamiltonian [Eq. (2)] is a constant of a given material when $\mathbf{R}$ is far away from the interface, when the averaging does not include the interface region. Let the interface be perpendicular to the $z$-direction and located in the $z=0$ plane. The "length" of the interface $L_{\text {if }}$ is determined by the region of space that consists of all $\mathbf{R}$-vectors such that the average $\left[u_{m}|V| u_{n}\right]_{\mathbf{R}}$ encompasses the interface region. In the case of the [001] interface $L_{\mathrm{if}}=a / 2$ and in the case of other interfaces considered in this work $L_{\text {if }}=a /(2 \sqrt{2})$, where $a$ is the lattice constant of the bulk.

Since the interface region is small and the envelope functions are slowly varying, the details of the variations of the $\left[u_{m}|V| u_{n}\right]_{\mathbf{R}}$ are not of primary importance; it is only the integral of this term over the interface region that determines its role in the envelope function Hamiltonian. In the flat interface model, the pseudopotentials are modeled to be equal to those of material A at one side of interface and moving sharply to those of material B at the other side of an interface. We then obtain

$$
\begin{aligned}
\int_{-L_{\mathrm{if}} / 2}^{+L_{\mathrm{if}} / 2}\left[u_{m}\left|H_{\mathrm{a}}\right| u_{n}\right]_{z_{0}} \mathrm{~d} z_{0}= & \int_{-L_{\mathrm{if}} / 2}^{+L_{\mathrm{if}} / 2}\left[u_{m}\left|\frac{p^{2}}{2 m_{0}}+V\right| u_{n}\right]_{z_{0}} \mathrm{~d} z_{0} \\
& -\left[u_{m}\left|\frac{p^{2}}{2 m_{0}}+\frac{V_{\mathrm{A}}+V_{\mathrm{B}}}{2}\right| u_{n}\right] L_{\mathrm{if}} \\
& +\left[u_{m}\left|\frac{p^{2}}{2 m_{0}}+V_{\mathrm{A}}\right| u_{n}\right] \frac{L_{\mathrm{if}}}{2}+\left[u_{m} \mid \frac{p^{2}}{2 m_{0}}\right. \\
& \left.+V_{\mathrm{B}} \mid u_{n}\right] \frac{L_{\mathrm{if}}}{2} .
\end{aligned}
$$


In the Eq. (3), the last two terms on the right hand side represent the bulk contribution to the Hamiltonian, while the first two terms are the interface contribution. This implies that for each interface, the envelope function Hamiltonian contains an additional term of the form $\Omega_{m n} \delta \theta(z)$, with $\Omega_{m n}$ given by the expression:

$$
\begin{aligned}
\Omega_{m n}= & \int_{-L_{\mathrm{if}} / 2}^{+L_{\mathrm{if}} / 2}\left[u_{m}\left|\frac{p^{2}}{2 m_{0}}+V\right| u_{n}\right]_{z_{0}} \mathrm{~d} z_{0} \\
& -\left[u_{m}\left|\frac{p^{2}}{2 m_{0}}+\frac{V_{\mathrm{A}}+V_{\mathrm{B}}}{2}\right| u_{n}\right] L_{\mathrm{if}} .
\end{aligned}
$$

When one chooses the bulk reference crystal as a virtual crystal being the "average" of crystals A and B, the last expression reduces to

$$
\Omega_{m n}=\int_{-L_{\mathrm{if}} / 2}^{+L_{\mathrm{if}} / 2}\left[u_{m}\left|\frac{p^{2}}{2 m_{0}}+V\right| u_{n}\right]_{z_{0}} \mathrm{~d} z_{0}-E_{m} \delta_{m n} L_{\mathrm{if}},
$$

where $E_{m}$ is the energy of band $m$ at the $\Gamma$ point. The actual interface matrices are given in Appendix A, while the crucial steps needed to evaluate the elements of these matrices are outlined in Sec. II of the supplementary information. ${ }^{65}$ The total interface Hamiltonian is then given by the equation

$$
\begin{aligned}
H_{\mathrm{if}}= & \Omega([001]) \delta \theta(z) \\
& +\Omega([101]) \delta \theta\left(\mathbf{r} \cdot \mathbf{n}_{1}-l\right)+\Omega([011]) \delta \theta\left(\mathbf{r} \cdot \mathbf{n}_{2}-l\right) \\
& +\Omega([\overline{1} 01]) \delta \theta\left(\mathbf{r} \cdot \mathbf{n}_{3}-l\right)+\Omega([0 \overline{1} 1]) \delta \theta\left(\mathbf{r} \cdot \mathbf{n}_{4}-l\right)
\end{aligned}
$$

In the above equation, $\delta \theta(z)$ function represents the delta function at a given interface, with an additional constraint that the function is nonzero only at the face of the pyramid. The vectors $\mathbf{n}_{i}$ are the unit vectors perpendicular to the faces of the pyramid and are given as $\mathbf{n}_{1}=1 / \sqrt{2} \cdot(1,0,1), \mathbf{n}_{2}=1 / \sqrt{2} \cdot(0,1,1)$, $\mathbf{n}_{3}=1 / \sqrt{2} \cdot(-1,0,1), \quad \mathbf{n}_{4}=1 / \sqrt{2} \cdot(0,-1,1), \quad$ and $l=$ $b /(2 \sqrt{2})$. The integrals needed to represent $H_{\text {if }}$ in the plane wave basis are given in Sec. IV in the supplementary material. $^{65}$

\section{RESULTS AND DISCUSSION}

To understand the role of interfaces and/or higher energy bands, we start by analyzing the conventional 8-band k·p Hamiltonian. We consider first its version in the absence of spin-orbit interaction. The symmetry group of such a Hamiltonian applied to a pyramidal square-based QD is the $C_{4 v}$ group. To show analytically that this is really the case, one needs to prove that the representations of the generators of the group, $D(g)$, commute with the Hamiltonian $H$, i.e., $[D(g), H]=0$. The generators of the $C_{4 v}$ group are the rotation by $\pi / 2$ about the $z$ axis, $R_{\pi / 2}$, and the reflection with respect to the $x=y$ plane $\sigma_{v}$. Equality of the operators $D(g) H$ and $H D(g)$ can be established by showing that they yield the same results when they act on any of the plane wave basis vectors that span the Hilbert space of the system. Indeed, we have shown that $\left[D\left(R_{\varphi}\right), H_{8 \mathbf{k} \cdot \mathbf{p}}\right]=0$ for $\varphi=n \pi / 2$ and $\left[D\left(\sigma_{v}\right)\right.$, $\left.H_{8 \mathbf{k} \cdot \mathbf{p}}\right]=0$ (see Sec. III of the supplementary material for a detailed proof). ${ }^{65}$

Several prominent features of the energy level structure [Table I(a)] and the wavefunctions [Fig. 1(a)] arise as a consequence of high symmetry of the system. The pairs of energy levels $\left(e_{1}, e_{2}\right),\left(h_{0}, h_{1}\right)$ and $\left(h_{4}, h_{5}\right)$ are degenerate. The presence of degenerate energy levels is a consequence of the fact that the $C_{4 v}$ group has a two dimensional representation $E$ (the notation of Ref. 71). Therefore, the states that transform according to that representation come in pairs and are degenerate. The high symmetry of the system can be also witnessed from the shape of the probability density isosurfaces [Fig. 1(a)]: All of them exhibit perfect $C_{4 v}$ symmetry.

Next, we include the spin-orbit interaction in the 8-band k·p Hamiltonian, case (b). The eigenstates now transform according to a representation of the double $C_{4 v}$ group which is a direct product of the representation of the single $C_{4 v}$ group and the representation $\mathcal{D}_{1 / 2}$ according to which the spin functions transform (Ref. 71, p. 142). If the representation obtained from the direct product is reducible, the inclusion of spin-orbit interaction leads to the removal of degeneracy of energy levels. Indeed, the product $E \times \mathcal{D}_{1 / 2}$ is equal to $E_{1}{ }^{\prime}+E_{2}{ }^{\prime}$ (the notation of Ref. 71). It is well understood that the effect of spin-orbit interaction on the states in the valence band is rather strong. Here we would, however, like to emphasize a less known fact that the spin-orbit interaction also causes the splitting of the $e_{1}$

\begin{tabular}{|c|c|c|c|c|c|c|c|c|c|}
\hline State & (a) & (b) & (c) & (d) & (e) & (f) & (g) & (h) & (i) \\
\hline e3 & 1.08163 & 1.06674 & 1.08878 & 1.30684 & 1.30772 & 1.03852 & 1.04622 & 1.30421 & 1.30463 \\
\hline e2 & 0.99336 & 0.96795 & 1.00170 & 1.25044 & 1.25570 & 0.97301 & 0.97747 & 1.24429 & 1.24514 \\
\hline e1 & 0.99336 & 0.96724 & 1.00074 & 1.23439 & 1.23943 & 0.97298 & 0.97746 & 1.22810 & 1.22900 \\
\hline $\mathrm{e} 0$ & 0.84346 & 0.81808 & 0.85115 & 1.12013 & 1.12543 & 0.83509 & 0.83754 & 1.11712 & 1.11768 \\
\hline h0 & -0.06722 & -0.03427 & -0.06475 & -0.05230 & -0.05052 & -0.06512 & -0.06495 & -0.05086 & -0.05066 \\
\hline h1 & -0.06722 & -0.03680 & -0.06698 & -0.06827 & -0.06699 & -0.06517 & -0.06500 & -0.06703 & -0.06673 \\
\hline h2 & -0.07389 & -0.03765 & -0.07248 & -0.07840 & -0.07843 & -0.07263 & -0.07231 & -0.07743 & -0.07717 \\
\hline h3 & -0.07883 & -0.04244 & -0.07708 & -0.09115 & -0.09046 & -0.07700 & -0.07675 & -0.08865 & -0.08827 \\
\hline h4 & -0.08518 & -0.04582 & -0.08119 & -0.10517 & -0.10565 & -0.08124 & -0.08117 & -0.10282 & -0.10241 \\
\hline h5 & -0.08518 & -0.04614 & -0.08360 & -0.10888 & -0.10859 & -0.08270 & -0.08242 & -0.10730 & -0.10698 \\
\hline
\end{tabular}
and $e_{2}$ states [shown in Table $\left.\mathrm{I}(\mathrm{b})\right]$. The existence of this splitting was established in Ref. 57 for pyramidal QDs and later on analyzed again in Ref. 72 for lens-shaped QDs. It

TABLE I. Energies (in eV) of top six hole energy levels and bottom four electron levels, for a square-based pyramidal InAs/GaAs QD with base width $b=100 \AA$, and base to height ratio $b / h=2$ calculated using different models. The letters in the first row in the table specify the model used in the calculation. 


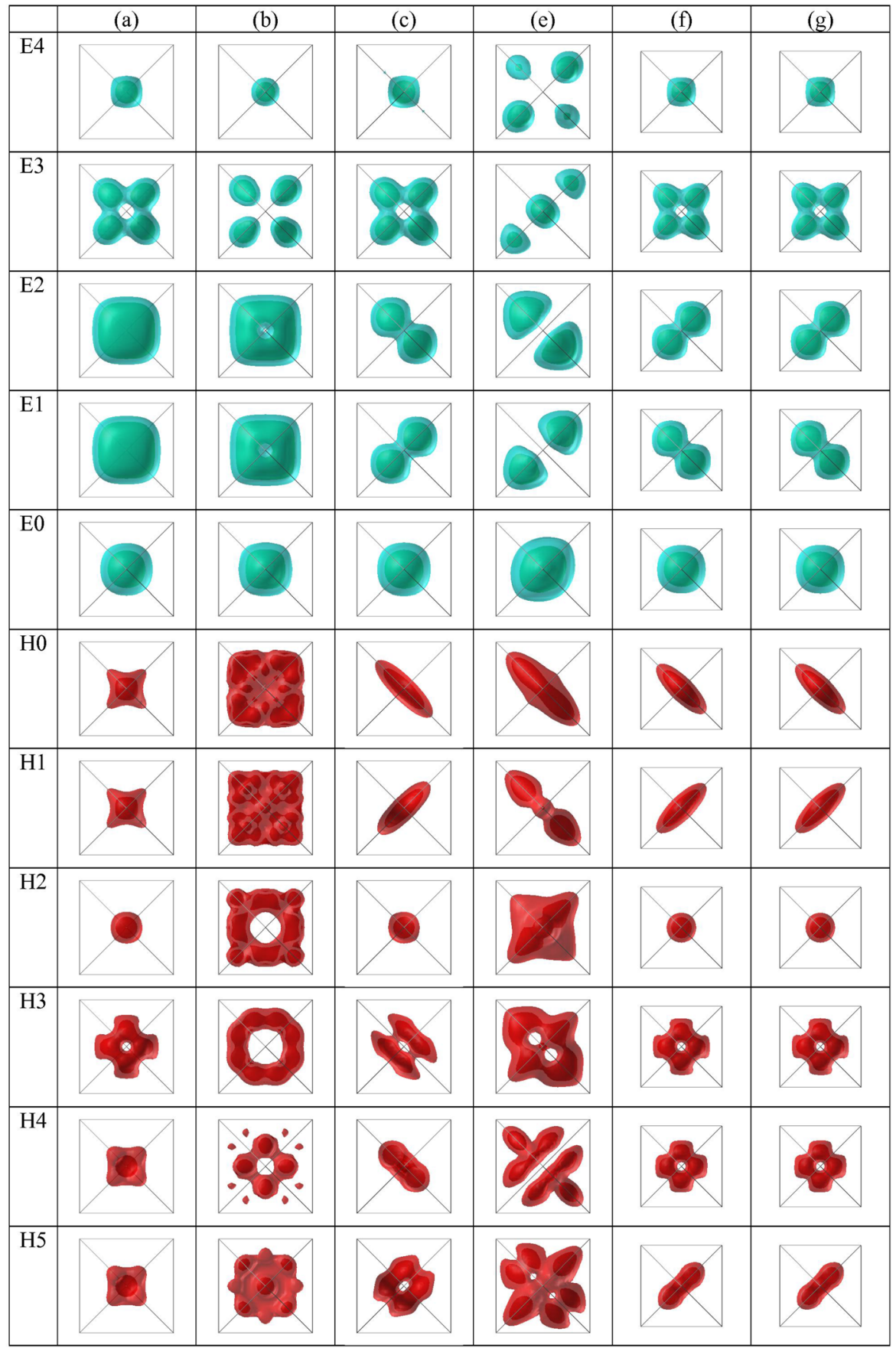

FIG. 1. (Color online) The wavefunctions squared for top six hole states and bottom five electron states for a square-based pyramidal InAs/GaAs QD with base width $b=100 \AA$, and base to height ratio $b / h=2$ calculated using different models. The letters (a)-(c), (e)-(g) specify the model used in the calculation. The isosurfaces are plotted at 25\% (transparent) and $75 \%$ of the maximal charge density.

is important to note here that this energy level splitting effect is not a signature of symmetry reduction. Indeed, it can be seen from Fig. 1(b) that the wavefunction moduli still exhibit a perfect $C_{4 v}$ symmetry.

We proceed to understand the role of interfaces on the energy level structure and the symmetry of the system, case (c). For that purpose we calculate the electronic structure of a QD using the 8-band k·p Hamiltonian in the absence of spin-orbit interaction and the presence of interface effects [Fig. 1(c) and Table I(c)]. Our analytical derivations, demonstrate that both the [001] interface and the joint effect of the other interfaces independently lead to the reduction of symmetry from $C_{4 v}$ to $C_{2 v}$ since both commutators $\left[D\left(R_{\varphi}\right)\right.$,
$\left.H_{[001]}\right]=0$ and $\left[D\left(R_{\varphi}\right), H_{\text {if }}\right]=0$ only if $\varphi=n \pi$ (see Sec. III of Ref. 65). The $C_{2 v}$ group has one dimensional irreducible representations only and cannot exhibit the doubly degenerate eigenstates. As a consequence, interface effects lead to splitting of the degenerate eigenstates by typically $1-3 \mathrm{meV}$, as shown in Table I(c). The low symmetry of the system can also be evidenced from the shape of the wavefunction isosurfaces. All the isosurfaces reduce their symmetry from $C_{4 v}$ [Fig. 1(a)] to $C_{2 v}$ [Fig. 1(c)]. The symmetry breaking is most pronounced for wavefunctions that were originally degenerate, while it is weaker, but still present, for the other ones.

To quantify the role of the dot size on the magnitude of interface induced effects, we compare the electronic 


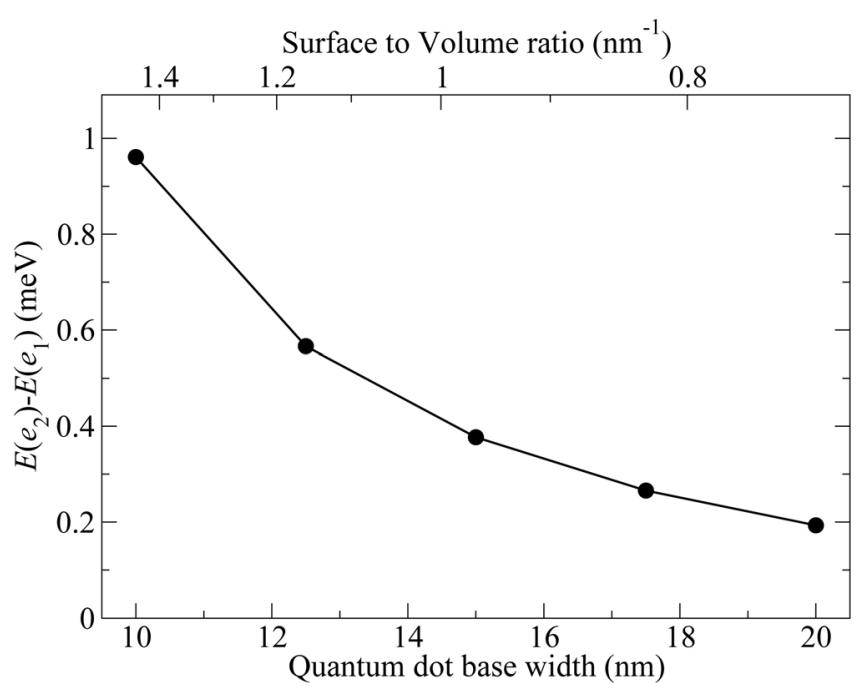

FIG. 2. The dependence of interface-induced splitting of the $e_{1}$ and $e_{2}$ levels on the size of an InAs/GaAs QD.

structure of the Hamiltonians (a) and (c) for several different QDs sizes. We use the splitting of the $e_{1}$ and $e_{2}$ states as a quantitative measure of interface effects. The results are summarized in Fig. 2. We find that the interface induced splitting of the $e_{1}$ and $e_{2}$ states decreases as the dot dimensions and the dot volume to surface ratio increase. The interface effects become less important for dots with large volume to surface ratio.

To identify the role of higher energy bands, we have extended the Hamiltonian to include 14 bands, case (f). We have found analytically that the 14 band k.p Hamiltonian commutes with the operators of the $C_{2 v}$ group representation, i.e., $\left[D\left(R_{\varphi}\right), H_{14 \mathbf{k} \cdot \mathbf{p}}\right]=0$ only for $\varphi=n \pi$ (see Sec. III of Ref. $65)$. The terms that contain the $P_{2}$ element which couples the top of the valence band, $\Gamma_{5 v}$, [that originates from the $p$ bonding states (denoted as $p_{b}$ ) of atoms in the bulk] with the second conduction band, $\Gamma_{5 c}$, [that originates from the $p$ antibonding states (denoted as $p_{a}$ ) of atoms in the bulk] are the only terms that prevent the $C_{4 v}$ symmetry. Therefore, the $P_{2}$ element can be identified as the symmetry breaking term in

TABLE II. Energies (in eV) of top six hole energy levels and bottom four electron levels, for a square-based pyramidal GaAs/AlGaAs QD with base width $b=100 \AA$, and base to height ratio $b / h=2$ calculated using different models. The letters in the first row in the table specify the model used in the calculation.

\begin{tabular}{lccccr}
\hline \hline State & (a) & (b) & (c) & (d) & (f) \\
\hline e3 & 1.87820 & 1.87153 & 1.87826 & 1.87158 & 1.87773 \\
e2 & 1.86355 & 1.85915 & 1.86428 & 1.85981 & 1.85947 \\
e1 & 1.86355 & 1.85866 & 1.86426 & 1.85932 & 1.85936 \\
e0 & 1.75534 & 1.75191 & 1.75641 & 1.75291 & 1.75234 \\
h0 & -0.07545 & -0.08834 & -0.07512 & -0.08818 & -0.07445 \\
h1 & -0.07545 & -0.09647 & -0.07551 & -0.09632 & -0.07461 \\
h2 & -0.08848 & -0.11788 & -0.08834 & -0.11756 & -0.08792 \\
h3 & -0.09860 & -0.12268 & -0.09519 & -0.12255 & -0.09346 \\
h4 & -0.10508 & -0.13360 & -0.09844 & -0.13340 & -0.09749 \\
h5 & -0.10508 & -0.14167 & -0.10486 & -0.14149 & -0.10347 \\
\hline \hline
\end{tabular}

the 14-band k·p Hamiltonian. This term introduces the splittings, Table I(f), which are however less pronounced than these of the interface terms; for example, the splitting of $e_{1}$ and $e_{2}$ is less than $0.1 \mathrm{meV}$. The effect of symmetry breaking on the wavefunctions is generally similar [see Fig. 1(f)] as in the case of interface-induced symmetry breaking. The inclusion of the additional band that gives the 16-band k·p Hamiltonian does not lead to any significant qualitative nor quantitative changes in the results, Table $\mathrm{I}(\mathrm{g})$.

It has been previously established ${ }^{57}$ that the piezoelectric effect also reduces the symmetry from $C_{4 v}$ to $C_{2 v}$. The results shown in Tables $\mathrm{I}(\mathrm{d}), \mathrm{I}(\mathrm{e}), \mathrm{I}(\mathrm{h})$ and $\mathrm{I}(\mathrm{i})$ indicate that splitting of $e_{1}$ and $e_{2}$ states induced by the piezoelectric effect is stronger than the splittings induced by other effects. One should also stress that in the absence of piezoelectric effect, the strain would also give rise to symmetry reduction if it were modeled using the Valence force field model. ${ }^{26,66-68,70}$

There is an interest to understand the role of other QD materials on the symmetry breaking and energy level splitting effects. We have therefore calculated the electronic structure of a QD based on a different material, namely, virtually strain free $\mathrm{GaAs} / \mathrm{Al}_{0.35} \mathrm{Ga}_{0.65} \mathrm{As} \mathrm{QD}$. This type of quantum dots cannot be realized via a conventional StrankiKrastanov growth technique which requires latticemismatched materials. Nevertheless, there has been an increasing interest in this quantum dot material system in recent years due to the introduction of techniques for controllable realization ${ }^{73-75}$ of such quantum dots. The energy levels of this dot are summarized in Table II. These results suggest that the splitting of $e_{1}$ and $e_{2}$ levels due to spin-orbit interaction and higher energy bands is of the similar order of magnitude as for InAs/GaAs. On the other hand, interface effects are much weaker in this material. This can be understood from the fact that AlGaAs and GaAs are more similar materials than InAs and GaAs.

\section{CONCLUSION}

In contrast to a popular belief that multiband envelope function $\mathbf{k} \cdot \mathbf{p}$ Hamiltonians cannot capture the right symmetry of QDs, we showed here the opposite. We put our conclusions into numbers for the case of square-based pyramidal QDs based on III-V materials with zinc blende crystal structure. We showed that the inclusion of interface band mixing effects leads to the reduction of symmetry from an artificial $C_{4 v}$, to the correct $C_{2 v}$. The main manifestation of interface effects are the energy level splittings between $\left(e_{1}, e_{2}\right),\left(h_{0}\right.$, $\left.h_{1}\right)$, and $\left(h_{4}, h_{5}\right)$ states of the order of $1-3 \mathrm{meV}$ in InAs/GaAs material system, which are much weaker in the AlGaAs/GaAs system. The splittings decrease as the dot size and consequently the volume to surface ratio increase. The inclusion of the additional bands beyond the standard 8 bands also leads to symmetry reduction to $C_{2 v}$, with splittings which are however weaker than the ones due to interfaces. We have found that that the lowest order multiband Hamiltonian whose kinetic part has the correct $C_{2 v}$ symmetry is the 14-band $\mathbf{k} \cdot \mathbf{p}$ Hamitonian. This symmetry reduction originates from the coupling between the top of the valence 
TABLE III. Relevant material parameters of the interfaces: InAs/GaAs and $\mathrm{GaAs} / \mathrm{Al}_{0.35} \mathrm{Ga}_{0.65} \mathrm{As}$

\begin{tabular}{lrc}
\hline \hline & InAs/GaAs & $\mathrm{GaAs} / \mathrm{Al}_{0.35} \mathrm{Ga}_{0.65} \mathrm{As}$ \\
\hline$a[\mathrm{eV} \AA]$ & 0.61220 & 0.14899 \\
$b[\mathrm{eV} \AA]$ & -0.36633 & 0.02861 \\
$c[\mathrm{eV} \AA]$ & -0.32427 & -0.07039 \\
$d[\mathrm{eV} \AA]$ & 0.02855 & 0.00008 \\
\hline \hline
\end{tabular}

$\left(\Gamma_{5 v}\right)$ and the second conduction $\left(\Gamma_{5 c}\right)$ band. The observed splittings are comparable to the ones that originate from spin-orbit coupling (these do not reduce the symmetry) and are much smaller than the ones from piezoelectric effect in strained systems. One can further note that other effects than the ones considered in this work can also affect the symmetry of the system, such as the atomic-scale randomness ${ }^{76}$ in QDs made of alloys, as well as the irregularity of the shape of the dot. Unfortunately, the mentioned symmetry reduction effects cannot be independently measured since in a given QD, they all act jointly. Despite that, our work provides a very important conceptual message: With appropriate treatment of relevant effects, the multiband envelope function Hamiltonians are fully capable of capturing the right symmetry of QD structures.

\section{ACKNOWLEDGMENTS}

S.T. thanks the STFC's UK e-Science SCARF facility for providing computer resources. N.V. was supported in part by the Ministry of Science and Technological Development of the Republic of Serbia, under project No. ON171017, and the European Commission under EU FP7 projects PRACE-1IP, HP-SEE and EGI-InSPIRE.

\section{APPENDIX A: INTERFACE HAMILTONIAN MATRICES}

To evaluate the $\Omega_{m n}$ terms, Eq. (5), we represent the pseudopotentials using the plane waves, where the form factors from Ref. 77 for InAs/GaAs, and Ref. 52 for $\mathrm{GaAs} / \mathrm{AlAs}$ are used. In the basis

$$
\left\{\left|s_{a}\right\rangle,\left|p_{x, b}\right\rangle,\left|p_{y, b}\right\rangle,\left|p_{z, b}\right\rangle\right\}
$$

we obtain the following expressions for the $\Omega_{m n}$ terms for the interfaces of interest in this paper:

$$
\begin{gathered}
\Omega([001])=\left(\begin{array}{cccc}
0 & 0 & 0 & -a \\
& 0 & b & 0 \\
& & 0 & 0 \\
& & & 0
\end{array}\right), \\
\Omega([101])=\left(\begin{array}{cccc}
0 & -c & 0 & -c \\
& 0 & d & 0 \\
& & 0 & d \\
& & & 0
\end{array}\right),
\end{gathered}
$$

$$
\begin{aligned}
& \Omega([011])=\left(\begin{array}{cccc}
0 & 0 & -c & -c \\
& 0 & d & d \\
& & 0 & 0 \\
& & & 0
\end{array}\right), \\
& \Omega([\overline{10} 1])=\left(\begin{array}{cccc}
0 & c & 0 & -c \\
& 0 & d & 0 \\
& & 0 & -d \\
& & & 0
\end{array}\right), \\
& \Omega([0 \overline{1} 1])=\left(\begin{array}{cccc}
0 & 0 & c & -c \\
& 0 & d & -d \\
& & 0 & 0 \\
& & & 0
\end{array}\right) .
\end{aligned}
$$

The values of the parameters $a, b, c$, and $d$ for the interfaces considered in this work are given in Table III.

${ }^{1}$ J. P. Reithmaier, G. Sek, A. Loffler, C. Hofmann, S. Kuhn, S. Reitzenstein, L. V. Keldysh, V. D. Kulakovskii, T. L. Reinecke, and A. Forchel, Nature 432, 197 (2004).

${ }^{2}$ Y. Léger, L. Besombes, J. Fernendez-Rossier, L. Maingault, and H. Mariette, Phys. Rev. Lett. 77, 107401 (2006).

${ }^{3}$ A. Zrenner, E. Beham, S. Stufler, F. Findeis, M. Bichler, and G. Abstreiter, Nature 418, 612 (2002).

${ }^{4}$ D. Pan, Y. P. Zeng, M. Y. Kong, J. Wu, Y. Q. Zhu, C. H. Zhang, J. M. Li, and C. Y. Wang, Electron. Lett. 32, 1726 (1996).

${ }^{5}$ D. Pan, E. Towe, and S. Kennerly, Appl. Phys. Lett. 73, 1937 (1998).

${ }^{6}$ S. Maimon, E. Finkman, G. Bahir, S. E. Schacham, J. M. Garcia, and P. M. Petroff, Appl. Phys. Lett. 73, 2003 (1998).

${ }^{7}$ P. Bhattacharya, S. Ghosh, and A. D. Stiff-Roberts, Annu. Rev. Mater. Res. 34, 1 (2004).

${ }^{8}$ N. Kirstaedter, N. N. Ledentsov, M. Grundmann, D. Bimberg, V. M. Ustinov, S. S. Ruvimov, M. V. Maximov, P. S. Kopev, Z. I. Alferov, U. Richter, P. Werner, U. Gosele, and J. Heydenreich, Electron. Lett. 30, 1416 (1994).

${ }^{9}$ A. Luque and A. Marti, Adv. Mater. 22, 160 (2010).

${ }^{10}$ A. Marti, E. Antolin, C. R. Stanley, C. D. Farmer, N. Lopez, P. Diaz, E. Canovas, P. G. Linares, and A. Luque, Phys. Rev. Lett. 97, 247701 (2006).

${ }^{11}$ S. A. Blokhin, A. V. Sakharov, A. M. Nadtochy, A. S. Pauysov, M. V. Maximov, N. N. Ledentsov, A. R. Kovsh, S. S. Mikhrin, V. M. Lantratov, S. A. Mintairov, N. A. Kaluzhniy, and M. Z. Shvarts, Semiconductors 43, 514 (2009).

${ }^{12}$ S. Tomić, T. S. Jones, and N. M. Harrison, Appl. Phys. Lett. 93, 263105 (2008).

${ }^{13}$ P. Michler, A. Kiraz, C. Becher, W. V. Schoenfeld, P. M. Petroff, L. D. Zhang, E. Hu, and A. Imamoglu, Science 290, 2282 (2000).

${ }^{14}$ C. Santori, M. Pelton, G. S. Solomon, Y. Dale, and Y. Yamamoto, Phys. Rev. Lett. 86, 1502 (2001).

${ }^{15}$ S. Kako, C. Santori, K. Hoshino, S. Gotzinger, Y. Yamamoto, and Y. Arakawa, Nat. Mater. 5, 887 (2006).

${ }^{16}$ S. Tomić and N. Vukmirović, Phys. Rev. B 79, 245330 (2009).

${ }^{17}$ N. Vukmirović and L.-W. Wang, Compr. Nanosci. Technol. 1, 189 (2010).

${ }^{18}$ J. Kim, L.-W. Wang, and A. Zunger, Phys. Rev. B 57, R9408 (1998).

${ }^{19}$ L. W. Wang, J. Kim, and A. Zunger, Phys. Rev. B 59, 5678 (1999).

${ }^{20}$ A. J. Williamson and A. Zunger, Phys. Rev. B 59, 15819 (1999).

${ }^{21}$ L.-W. Wang and A. Zunger, Phys. Rev. B 59, 15806 (1999).

${ }^{22}$ T. Saito, J. N. Schulman, and Y. Arakawa, Phys. Rev. B 57, 13016 (1998).

${ }^{23}$ S. Lee, J. Kim, L. Jönsson, J. W. Wilkins, G. W. Bryant, and G. Klimeck, Phys. Rev. B 66, 235307 (2002).

${ }^{24}$ R. Santoprete, B. Koiller, R. B. Capaz, P. Kratzer, Q. K. K. Liu, and M. Scheffler, Phys. Rev. B 68, 235311 (2003).

${ }^{25}$ W. Sheng, S.-J. Cheng, and P. Hawrylak, Phys. Rev. B 71, 035316 (2005).

${ }^{26}$ O. Stier, M. Grundmann, and D. Bimberg, Phys. Rev. B 59, 5688 (1999).

${ }^{27}$ C. Pryor, Phys. Rev. Lett. 80, 3579 (1998).

${ }^{28}$ H. Jiang and J. Singh, Phys. Rev. B 56, 4696 (1997).

${ }^{29}$ C. Pryor, Phys. Rev. B 57, 7190 (1998).

${ }^{30}$ S. Tomić, A. G. Sunderland, and I. J. Bush, J. Mater. Chem. 16, 1963 (2006). 
${ }^{31}$ G. Bester and A. Zunger, Phys. Rev. B 71, 045318 (2005).

${ }^{32}$ P. von Allmen, Phys. Rev. B 46, 15382 (1992).

${ }^{33}$ U. Rössler, Solid State Comm. 49, 943 (1984).

${ }^{34}$ H. Mayer and U. Rössler, Phys. Rev. B 44, 9048 (1991).

${ }^{35}$ C. Hermann and C. Weisbuch, Phys. Rev. B 15, 823 (1977).

${ }^{36}$ P. Pfeffer and W. Zawadzki, Phys. Rev. B 53, 12813 (1996).

${ }^{37}$ P. Pfeffer and W. Zawadzki, Phys. Rev. B 41, 1561 (1990).

${ }^{38}$ M. Cardona, N. E. Christensen, and G. Fasol, Phys. Rev. B 38, 1806 (1988).

${ }^{39}$ S. B. Radhia, S. Ridene, K. Boujdaria, H. Bouchriha, and G. Fishman, J. Appl. Phys. 92, 4422 (2002).

${ }^{40}$ S. B. Radhia, K. Boujdaria, S. Ridene, H. Bouchriha, and G. Fishman, J. Appl. Phys. 94, 5726 (2003).

${ }^{41}$ O. Zitouni, K. Boujdaria, and H. Bouchriha, Semicond. Sci. Technol. 20, 908 (2005).

${ }^{42}$ M. Cardona and F. H. Pollak, Phys. Rev. 142, 530 (1966).

${ }^{43}$ N. Fraj, I. Saïdi, S. B. Radhia, and K. Boujdaria, J. Appl. Phys. 102, 053703 (2007)

${ }^{44}$ C. Bulutay, Turk. J. Phys. 30, 287 (2006).

${ }^{45}$ D. Rideau, M. Feraille, L. Ciampolini, M. Minondo, C. Tavernier, H. Jaouen, and A. Ghetti, Phys. Rev. B 74, 195208 (2006).

${ }^{46}$ S. Richard, F. Aniel, and G. Fishman, Phys. Rev. B 70, 235204 (2004).

${ }^{47}$ M. E. Kurdi, G. Fishman, S. Sauvage, and P. Boucaud, J. Appl. Phys. 107, 013710 (2010).

${ }^{48}$ I. Saïdi, S. B. Radhia, and K. Boujdaria, J. Appl. Phys. 104, 023706 (2008).

${ }^{49}$ I. Saïdi, S. B. Radhia, and K. Boujdaria, J. Appl. Phys. 107, 043701 (2010).

${ }^{50}$ U. Rössler and J. Kainz, Solid State Comm. 121, 313 (2002).

${ }^{51}$ J. P. Cuypers and W. van Haeringen, Phys. Rev. B 48, 11469 (1993).

${ }^{52}$ J.-B. Xia, Phys. Rev. B 41, 3117 (1990).

${ }^{53}$ P. C. Klipstein, Phys. Rev. B 81, 235314 (2010).

${ }^{54}$ B. A. Foreman, Phys. Rev. Lett. 81, 425 (1998).

${ }^{55}$ C. R. Pidgeon and R. N. Brown, Phys. Rev. 146, 575 (1966).

${ }^{56}$ T. B. Bahder, Phys. Rev. B 41, 11992 (1990).

${ }^{57}$ N. Vukmirović, D. Indjin, V. D. Jovanović, Z. Ikonić, and P. Harrison, Phys. Rev. B 72, 075356 (2005).

${ }^{58}$ N. Vukmirović, Z. Ikonić, D. Indjin, and P. Harrison, J. Phys.: Condens. Matter 18, 6249 (2006)
${ }^{59}$ N. Vukmirović and S. Tomić, J. Appl. Phys. 103, 103718 (2008).

${ }^{60}$ S. Dalessi and M.-A. Dupertuis, Phys. Rev. B 81, 125106 (2010).

${ }^{61}$ A. Zunger, Phys. Stat. Sol. A 190, 467 (2002).

${ }^{62}$ M. A. Cusack, P. R. Briddon, and M. Jaros, Phys. Rev. B 54, R2300 (1996).

${ }^{63}$ G. Bester, X. Wu, D. Vanderbilt, and A. Zunger, Phys. Rev. Lett. 96, 187602 (2006).

${ }^{64}$ A. Schliwa, M. Winkelnkemper, and D. Bimberg, Phys. Rev. B 76, 205324 (2007).

${ }^{65}$ See supplementary material at http://dx.doi.org/10.1063/1.3631048 for the expressions for Hamiltonians used in this work (Sec. I), the derivation of the crucial matrix element for the evaluation of the interface term in the Hamiltonian (Sec. II), proofs of symmetry of the Hamiltonians (Sec. III) and the expressions for integrals of plane waves over the surfaces of the QD (Sec. IV).

${ }^{66}$ D. Bimberg, M. Grundmann, and N. N. Ledentsov, Quantum Dot Heterostructures (John Wiley, Chichester, U.K., 1999).

${ }^{67}$ O. Stier, Electronic and Optical Properties of Quantum Dots and Wires (Wissenschaft and Technik Verlag, Berlin, 2000).

${ }^{68}$ C. Pryor, J. Kim, L. W. Wang, A. J. Williamson, and A. Zunger, J. Appl. Phys. 83, 2548 (1998).

${ }^{69}$ A. D. Andreev, J. R. Downes, D. A. Faux, and E. P. O’Reilly, J. Appl. Phys. 86, 297 (1999).

${ }^{70}$ M. Tadić, F. M. Peeters, K. L. Janssens, M. Korkusiński, and P. Hawrylak, J. Appl. Phys. 92, 5819 (2002).

${ }^{71}$ G. L. Bir and G. E. Pikus, Symmetry and Strain-Induced Effects in Semiconductors (Wiley, New York, 1974).

${ }^{72}$ J. Even, F. Doré, C. Cornet, and L. Pedesseau, Phys. Rev. B 77, 085305 (2008).

${ }^{73}$ N. K. K. Watanabe and Y. Gotoh, Jpn. J. Appl. Phys. Part 2 39, L79 (2000).

${ }^{74}$ A. Rastelli, S. Stufler, A. Schliwa, R. Songmuang, C. Manzano, G. Costantini, K. Kern, A. Zrenner, D. Bimberg, and O. G. Schmidt, Phys. Rev. Lett. 92, 166104 (2004).

${ }^{75}$ D. P. Kumah, S. Shusterman, Y. Paltiel, Y. Yacoby, and R. Clarke1, Nat. Nanotechnol. 4, 835 (2009).

${ }^{76}$ V. Mlinar and A. Zunger, Phys. Rev. B 79, 115416 (2009).

${ }^{77}$ See http://www.research.ibm.com/DAMOCLES/html_files/numerics.html\# table1 for InAs/GaAs. 